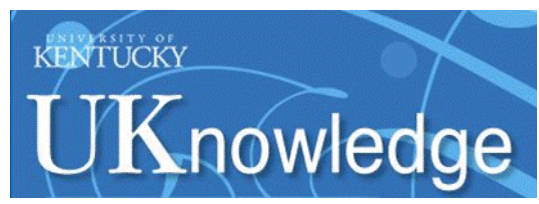

University of Kentucky

UKnowledge

Mechanical Engineering Faculty Publications

Mechanical Engineering

4-9-2018

\title{
Scalable Patterning Using Laser-Induced Shock Waves
}

\author{
Saidjafarzoda Ilhom \\ Western Kentucky University \\ Khomidkhodza Kholikov \\ Western Kentucky University \\ Peizhen Li \\ University of Kentucky, peizhen.li@uky.edu \\ Claire Ottman \\ Western Kentucky University \\ Dylan Sanford \\ Western Kentucky University
}

See next page for additional authors

Follow this and additional works at: https://uknowledge.uky.edu/me_facpub

Part of the Mechanical Engineering Commons, and the Semiconductor and Optical Materials

Commons

Right click to open a feedback form in a new tab to let us know how this document benefits you.

\section{Repository Citation}

Ilhom, Saidjafarzoda; Kholikov, Khomidkhodza; Li, Peizhen; Ottman, Claire; Sanford, Dylan; Thomas, Zachary; San, Omer; Karaca, Haluk E.; and Er, Ali O., "Scalable Patterning Using Laser-Induced Shock Waves" (2018). Mechanical Engineering Faculty Publications. 41.

https://uknowledge.uky.edu/me_facpub/41

This Article is brought to you for free and open access by the Mechanical Engineering at UKnowledge. It has been accepted for inclusion in Mechanical Engineering Faculty Publications by an authorized administrator of UKnowledge. For more information, please contact UKnowledge@lsv.uky.edu. 


\section{Scalable Patterning Using Laser-Induced Shock Waves}

Digital Object Identifier (DOI)

https://doi.org/10.1117/1.0E.57.4.041413

\section{Notes/Citation Information}

Published in Optical Engineering, v. 57, no. 4, article 041413, p. 1-6.

Saidjafarzoda Ilhom, Khomidkhodza Kholikov, Peizhen Li, Claire Ottman, Dylan Sanford, Zachary Thomas, Omer San, Haluk E. Karaca, Ali O. Er, "Scalable patterning using laser-induced shock waves", Optical Engineering 57(4), 041413 (April 9, 2018). DOI: https://doi.org/10.1117/1.0E.57.4.041413

(c) 2018 Society of Photo Optical Instrumentation Engineers (SPIE). One print or electronic copy may be made for personal use only. Systematic reproduction and distribution, duplication of any material in this paper for a fee or for commercial purposes, or modification of the content of the paper are prohibited.

The copyright holder has granted the permission for posting the article here.

\section{Authors}

Saidjafarzoda Ilhom, Khomidkhodza Kholikov, Peizhen Li, Claire Ottman, Dylan Sanford, Zachary Thomas, Omer San, Haluk E. Karaca, and Ali O. Er 


\section{Optical Engineering}

\section{Scalable patterning using laser- induced shock waves}

Saidjafarzoda Ilhom

Khomidkhodza Kholikov

Peizhen Li

Claire Ottman

Dylan Sanford

Zachary Thomas

Omer San

Haluk E. Karaca

Ali O. Er 


\title{
Scalable patterning using laser-induced shock waves
}

\author{
Saidjafarzoda Ilhom, ${ }^{\text {a }}$ Khomidkhodza Kholikov, ${ }^{a}$ Peizhen Li, ${ }^{\text {b }}$ Claire Ottman, ${ }^{\text {a }}$ Dylan Sanford, ${ }^{a}$ Zachary Thomas, ${ }^{a}$ \\ Omer San, ${ }^{\mathrm{c}}$ Haluk E. Karaca, ${ }^{\mathrm{b}}$ and Ali O. Era ${ }^{\mathrm{a}}$ \\ ${ }^{a}$ Western Kentucky University, Department of Physics and Astronomy, Bowling Green, Kentucky, United States \\ bUniversity of Kentucky, Department of Mechanical Engineering, Lexington, Kentucky, United States \\ 'Oklahoma State University, School of Mechanical and Aerospace Engineering, Stillwater, Oklahoma, United States
}

\begin{abstract}
An advanced direct imprinting method with low cost, quick, and minimal environmental impact to create a thermally controllable surface pattern using the laser pulses is reported. Patterned microindents were generated on $\mathrm{Ni}_{50} \mathrm{Ti}_{50}$ shape memory alloys and aluminum using an $\mathrm{Nd}$ : YAG laser operating at $1064 \mathrm{~nm}$ combined with a suitable transparent overlay, a sacrificial layer of graphite, and copper grid. Laser pulses at different energy densities, which generate pressure pulses up to a few GPa on the surface, were focused through the confinement medium, ablating the copper grid to create plasma and transferring the grid pattern onto the surface. Scanning electron microscope and optical microscope images show that various patterns were obtained on the surface with high fidelity. One-dimensional profile analysis indicates that the depth of the patterned sample initially increases with the laser energy and later levels off. Our simulations of laser irradiation process also confirm that high temperature and high pressure could be generated when the laser

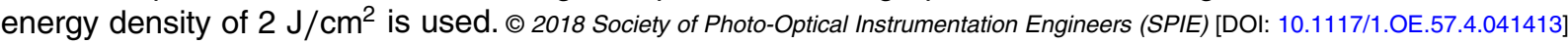

Keywords: laser shock wave; patterned surfaces; aluminum; shape memory alloys.

Paper 171585SS received Oct. 6, 2017; accepted for publication Mar. 13, 2018; published online Apr. 9, 2018.

\section{Introduction}

The development of micro/nanoscale patterning onto metallic components is of great significance in aerospace, microelectronics, and biomedical sectors ${ }^{\mathbb{Q}}$ Recently, there have been numerous techniques under investigation in these fields. Lithography-related routes, such as nanoimprint and step and flash imprint, employed to achieve submicron/nanoimprints, but are limited only to polymeric materials. $\square$ Moreover, these methods suffer from several drawbacks such as high cost, complex procedures, low reproducibility and throughput, and adverse environmental effects. $[$ Laser methods have opened a way of fabricating periodic structures directly on polymers, ceramics, metals, and on a variety of metallic components

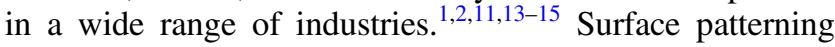
by lasers offers a variety of advantages such as flexibility, cleanliness, precise modification of the surface, remote and contact-free operation, precise energy deposition, and scalability $\square$ Unlike the conventional patterning methods, the laser patterning is inexpensive, environmentally friendly, faster than former with the minimal distortion, and does not involve any heating or etching process $\square$ Examples of laserpatterning techniques include laser shock peening, laser shock forming, and laser direct-write (also known as laser scribing)

In laser shock peening, a high energy laser beam is directed onto an ablative layer placed between material and dielectric medium, which serves as a tamping medium for the laser-generated shock wave ${ }^{\mathbb{9}}$ Consequently, the shock wave results in high compressive residual stress due to severe plastic deformation, which has been reported to significantly increase the fatigue life of materials used in a wide range of industries.
Similarly, in laser-assisted direct-write, a high power laser pulse directly interacts with a thin layer of material. Laser direct-write allows precise control of material properties with high resolution and complex structure with relative easiness. Laser pulses can induce high amplitude plasma pressure $(>1 \mathrm{GPa}$ ) in a short duration (usually on the order of $10^{-9} \mathrm{~s}$ ) that creates an intense shock wave, and con-

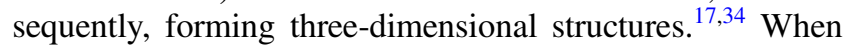
an additional transparent overlay is used, enhanced pressure (4 to 10 times higher) on the material surface could be attained and gas breakdown loss be prevented 6 [10

In laser-induced shock wave direct imprinting, expansion of plasma propels the underlying grid material into samples, creating patterned microindents. 2 B Even a single laser pulse can create pattern once the power density exceeds a certain threshold. Laser shock wave imprinting offers a variety of advantages where any template can be transferred onto the desired surface with high fidelity. Patterning of the predetermined template is highly scalable and the size and shape of the template allow precise control. These characteristics make laser-induced shock wave-assisted imprinting highly desirable in the patterning of functional devices especially in aerospace applications.

The laser absorption depth and the amount of material removed by a single-laser pulse depend on the material's optical properties and laser parameters. The properties of laser-produced plasma, such as the degree of ionization and temperature of plasma species can evolve quickly and sharply that depend on various parameters, such as the laser wavelength, energy density, repetition rate, pulse duration, the spot size on target, target composition, and surface quality $\left.{ }^{\mathrm{B}} \mathrm{B}\right]$ The laser pulse energy is initially absorbed predominantly by the surface electrons, which lead to a sharp 
temperature gradient in penetration depth and plasma formation. The material experiences a phase transformation from solid to vapor; thus a pressure (shock) wave is generated and propagates through the depth of the sample. The energy is redistributed between the energy of plume and shock waves during expansion, which is usually around $100 \mathrm{~ns}$. $\$ 0$

As shock wave travels through a substrate, plastic deformations occur to a depth where the peak pressure drops below the Hugoniot elastic limit. Amount of deformation depends on the pressure. Therefore, it is important to estimate the peak pressure, which is given as

$$
P(\mathrm{GPa})=0.01\left[\frac{Z\left(\mathrm{~g} \mathrm{~cm}^{-2} \mathrm{~s}^{-1}\right) I_{0}\left(\mathrm{GW} / \mathrm{cm}^{2}\right) \delta}{2 \delta+3}\right]^{1 / 2},
$$

where $I_{o}$ is the incident laser power density, $P$ is the pressure, and $Z$ is the reduced acoustic impedance between a target and medium, and $\delta$ is the efficiency of plasma material interaction.

Shape memory alloys (SMAs) can produce very high actuation strain (8\% uniaxial strain), stress ( 400 MPa), and work output $\left(\sim 10 \mathrm{MJ} / \mathrm{m}^{3}\right)$ as a result of reversible martensitic phase transformations. 20 SMAs are also being used in aerospace industry for decades as hydraulic tubing coupling, actuators for hingeless ailerons, wing twisting, tailoring the inlet geometry and orientation of propulsion systems, changing fan/nozzle area, noise mitigation at takeoff and landing, rotorcrafts, low-shock release devices, and optimizing dynamic properties of aircraft panels. Institute of Aeronautics and Astronautics (AIAA) named SMAs as one of the 10 emerging aerospace technologies. Among these applications, morphing at micro- and macroscales attracts considerable interest 40 Laser patterning on SMAs will generate controllable surfaces since SMAs are a class of active materials that can change their shape reversibly with a change in temperature, stress, or magnetic field. Surface roughness and texture can drastically alter the characteristics of turbulent flow, which lowers the drag and friction forces exerted on the surface of a moving object in a fluid. It has been reported that the "riblets" on the shark skin can reduce the wall shear stress or friction up to $10 \%$ [5 1 wile many surface structures are known to increase friction and drag force. Consequently, it may be possible to optimize the flight characteristics of moving objects by reversibly controlling surface roughness and texture. For micromorphing, shape memory surfaces can be produced by embedding SMAs into aircraft structure and forming "hybrid structures., 25 The temperature change (e.g., triggered by electrical current) will activate SMAs and surface geometry will change upon phase transformation where transformed surfaces could generate very different drag or friction forces that can be optimized for aircraft operation. SMAs can produce reversible surface protrusions based on the phase change between martensite and austenite 56 Thermal cycling which may be repeated indefinitely results in a "bumpy" or flat surface.

In this study, we have patterned aluminum and NiTi SMA with different templates. We have also analyzed the changes in depth of the generated patterns on NiTi SMA with respect to the laser energy density and reported optimum conditions to generate smooth patterns with the highest depth.

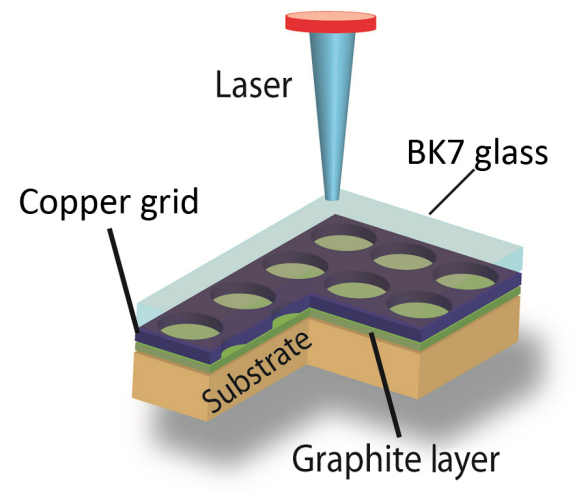

Fig. 1 Illustration of laser-induced shockwave imprinting.

\section{Experimental Conditions}

Aluminum was obtained from a commercially available source. The alloys were first electrical-discharge machined to a circular plate with a diameter of $10 \mathrm{~mm}$ and thickness of $1 \mathrm{~mm}$. The surface roughness of samples was reduced to $0.05 \mu \mathrm{m}$ in five steps by using Buehler EcoMet 250 GrinderPolisher with an AutoMet 250 Power head. Transformation temperatures were determined using a Perkin-Elmer Pyris 1 differential scanning calorimeter. The martensite and austenite start and finish temperatures $\left(\mathrm{M}_{\mathrm{s}}, \mathrm{M}_{\mathrm{f}}, \mathrm{A}_{\mathrm{s}}\right.$, and $\mathrm{A}_{\mathrm{f}}$, respectively) are $78^{\circ} \mathrm{C}, 45^{\circ} \mathrm{C}, 85^{\circ} \mathrm{C}, 122^{\circ} \mathrm{C}$. Samples were patterned by the nanosecond $\mathrm{Nd}$ :YAG-pulsed laser Continuum Surelite II. Laser has a fundamental wavelength of $1064 \mathrm{~nm}$, pulse width of $5 \mathrm{~ns}, 10 \mathrm{~Hz}$ repetition rate, and Gaussian shape with $6 \mathrm{~mm}$ diameter of an unfocused beam (measured at $\left.1 / \mathrm{e}^{2}\right)$. The experimental setup for pulsed laser-assisted shock wave imprinting in our lab is shown in Fig. 11.

A thin layer of graphite was sprayed as a sacrificial layer to increase the pressure and to protect the material from damage caused by ablation and melting 10 On top of the graphite layer, a copper grid (SPI Supplies) was used as both ablative material and punch. A piece of BK7 glass and water was used to confine the plasma generated by nanosecond laser. As a result, prepared samples were irradiated by laser energy densities between 1.3 and $3.2 \mathrm{~J} / \mathrm{cm}^{2}$ with a beam diameter of $3 \mathrm{~mm}$. The expansion of copper plasma was confined by confinement medium, mainly glass, which pushed the grid into the surface. This highly dynamic force creates plastic deformation on the surface leading to a surface pattern which is similar to the hole of the copper grid.

After irradiation, the copper grid was peeled off and the graphite layer was washed off with acetone. Morphological properties of the surface were investigated by scanning electron microscope (SEM) (Jeol 6510LV) and a light microscope (Keyence VHX500F). Laser parameters were adjusted to optimize the protrusion heights.

\section{Results and Discussions}

Figure 2 shows successful imprinting of hexagonal copper grid onto an aluminum sample with a single pulse with the energy density of $6.4 \mathrm{~J} / \mathrm{cm}^{2}$. Aluminum samples have a smooth surface after imprinting. The hexagonal grid has 698 lines/inch, hole width of $29 \mu \mathrm{m}$, pitch width of $37 \mu \mathrm{m}$, and bar width of $8 \mu \mathrm{m}$ with diameter of $3 \mathrm{~mm}$. 


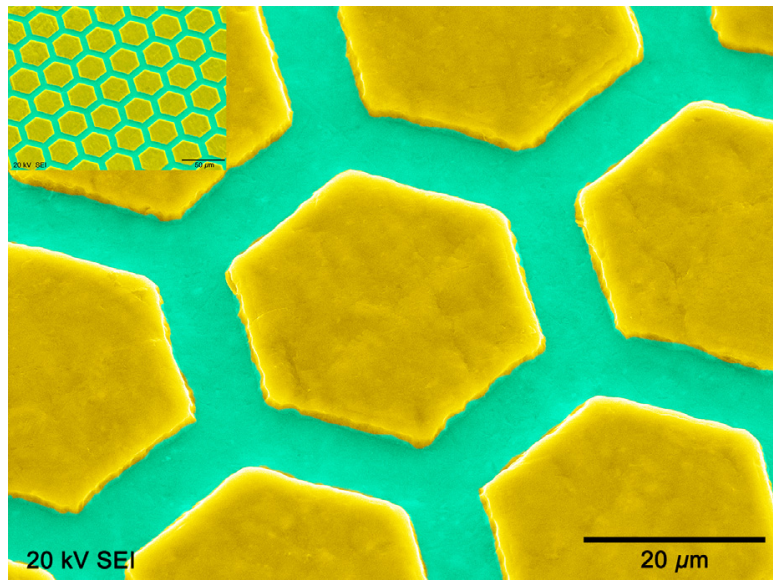

Fig. 2 The SEM image of the patterning on aluminum with hexagonal shapes.

After irradiation, a patterned surface with high fidelity is visible with optical microscope and SEM. The inset shows the larger area of the patterned surface.

Figure 3 shows another patterning on the aluminum sample with square copper grids. The fine square grid has 2000 lines/inch, pitch width of $12.5 \mu \mathrm{m}$, hole width $7.5 \mu \mathrm{m}$, and bar width of $5 \mu \mathrm{m}$ with diameter of $3 \mathrm{~mm}$. It is evident that the geometry of the grids can be transferred onto the sample with high precisions. The laser beam can be scanned over the surface to produce the desired pattern at a larger scale. By changing the number of pulses at each point and laser fluence, depth of the pattern can be controlled precisely.

Figure 7 shows the patterning on the NiTi SMAs when a laser pulse with fluence of $2 \mathrm{~J} / \mathrm{cm}^{2}$ is used. The square grid has 300 lines/inch, hole width $58 \mu \mathrm{m}$, pitch width of $83 \mu \mathrm{m}$, and bar width of $25 \mu \mathrm{m}$ with diameter of $3 \mathrm{~mm}$ and thickness of $20 \mu \mathrm{m}$. SMAs recover their previously defined shape after deformation once they are heated to a certain temperature. This is known as shape memory recovery effect (SME) and is due to stress-induced martensitic phase transformation 100 Upon deformation, the reversible twoway shape memory effect (TWSME) could be obtained. TWSME on NiTi surface can be induced using an indentation technique, a technique where indenter is pushed against

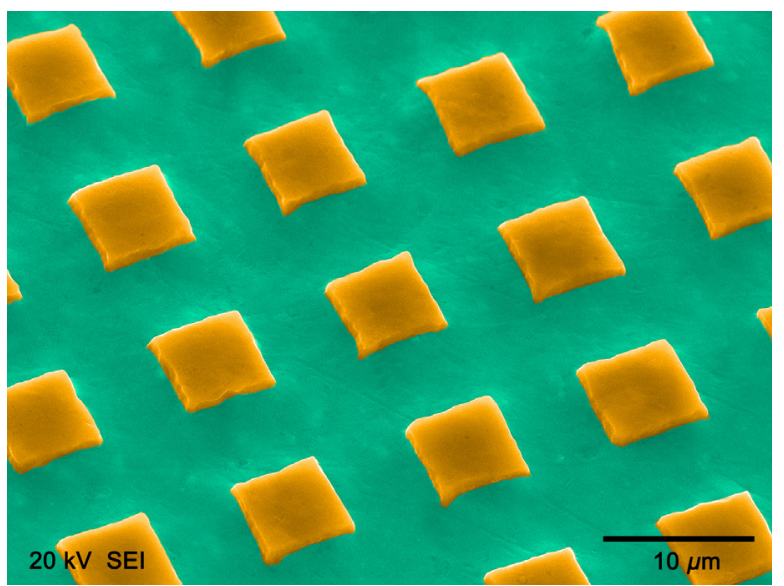

Fig. 3 The patterning on aluminum with square shapes.

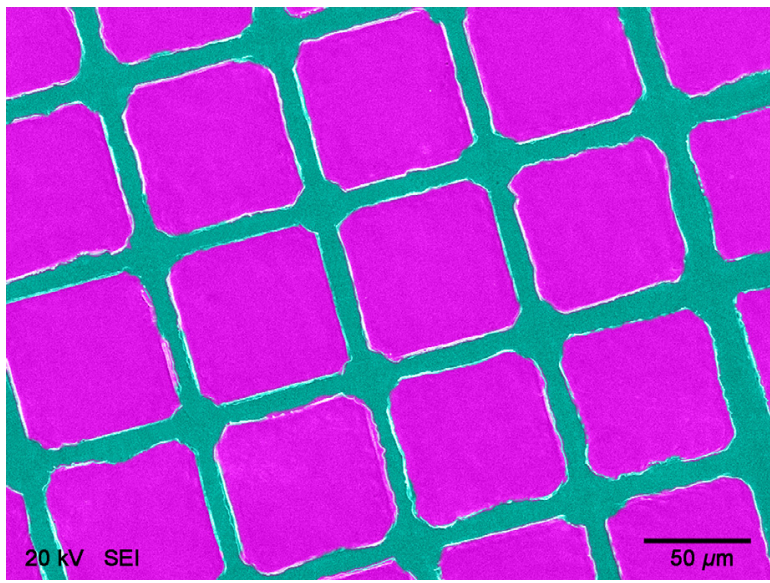

Fig. 4 Patterning on NiTi SMAs at $2.0 \mathrm{~J} / \mathrm{cm}^{2}$ energy.

the material to promote large-stress-induced transformation. 3 回回 In this study, we also show that laser-induced shock waves can be used for patterning and as well as obtaining TWSME.

Figure 5 shows the line scan of the patterned surface obtained with $2 \mathrm{~J} / \mathrm{cm}^{2}$. The average depth of the indent is around $1.5 \mu \mathrm{m}$. As expected, patterns that were generated on the surface have the similar dimensions as the copper grid.

In order to investigate and find the optimum laser parameters to achieve indents with high recovery ratio, patterns with different depths were obtained by changing the laser fluence between 1.3 and $3.2 \mathrm{~J} / \mathrm{cm}^{2}$. Figure 6 shows the change of the pattern's depth generated on the surface with respect to laser energy density. These depth measurements are the average depth of eight different points from three separate samples being irradiated by 1.3 to $3.2 \mathrm{~J} / \mathrm{cm}^{2}$ energies.

The depth increases when the laser fluence increases. After around $2.65 \mathrm{~J} / \mathrm{cm}^{2}$, depth of the patterns is leveled off. It is well known that above the ablation threshold, thickness or volume of material removed per pulse typically shows a logarithmic increase with fluence according to the Beer-Lambert law. Increasing the laser energy increases the kinetic energy of the particles being ejected from the target and the amount of material removed. The laser energy as

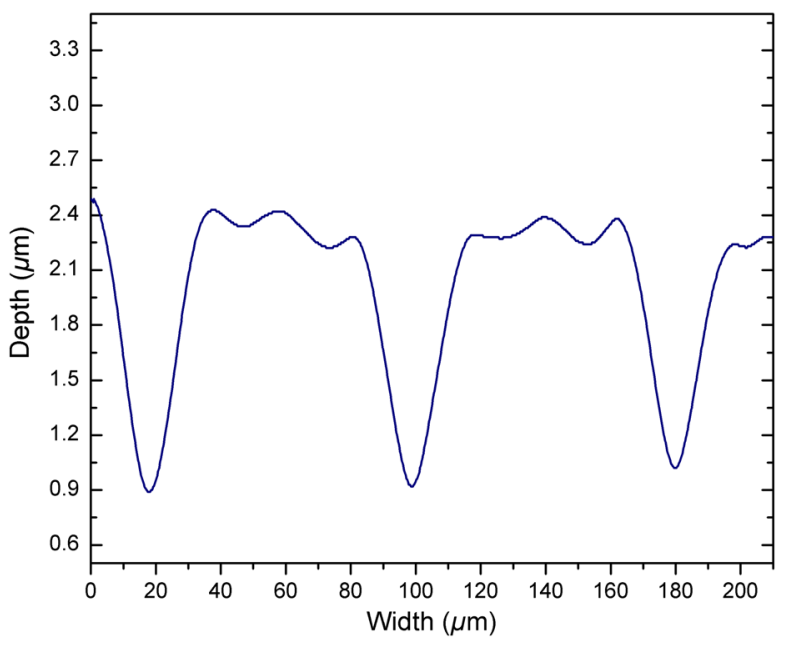

Fig. 5 Linear height profile of patterns on NiTi at $2.0 \mathrm{~J} / \mathrm{cm}^{2}$ energy. 


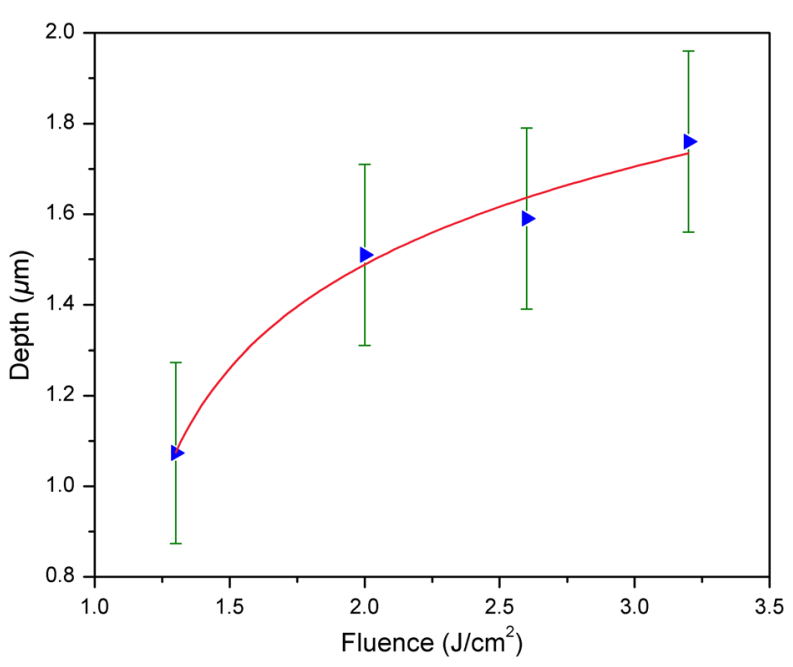

Fig. 6 Indent depth versus laser energy on NiTi SMAs.

a control parameter is known to significantly influence the morphology of the pattern and cause changes in the structure.

To determine the recovery of patterned SMA surfaces, samples were heated to $160^{\circ} \mathrm{C}$ and their depth profile and maximum depth were measured. Linear depth profiles were measured at four different positions to get better reliability. Upon the determination of maximum indent depth at $160^{\circ} \mathrm{C}$, the recovery rate (the difference between hot and cold state over cold state) was calculated as a function of energy and duration. Our results show that recovery ratio of $30 \%$ can be obtained when laser energy density was $0.18 \mathrm{~J} / \mathrm{cm}^{2}$ and irradiation time was $1 \mathrm{~s}$ with total 10 pulses. The recovery ratio subsequently decreased to $15 \%$ with increasing depth.

Laser energy was shown to play a significant role in surface patterning. Patterns generated on NiTi SMAs will allow creating a controllable surface by temperature change (e.g., triggered by electrical current), which will activate SMAs and surface geometry will change upon phase transformation. Thus, transformed surfaces could generate very different drag or friction forces that can be optimized for aircraft operation. Further analysis is needed to find the optimum laser properties for recovery of the patterns and the influence of the shapes of the created patterns on friction and drag reduction.

\subsection{Simulation of Laser Absorption on Copper Surface}

It is well known that lasers can produce high temperature and high pressure when absorbed by a material. In most of the practical applications, it is critical to determine the heat transfer mechanism for the accuracy of laser processing. To better understand the pressure propagation, a numerical simulation of pressure evolution in copper irradiated by a nanosecond pulsed laser (1064 nm wavelength, $10 \mathrm{~Hz}, 2 \mathrm{~J} / \mathrm{cm}^{2}, 3 \mathrm{~mm}$ beam diameter, and $5 \mathrm{~ns}$ pulse width) was conducted using the fifth-order weighted essentially nonoscillatory (WENO) finite volume method 65 coupled with a Mie-Gruneisen model 6 The Gruneisen constants are set as $s_{0}=1.249$, $\Gamma_{0}=1.99(T \leq 700 \mathrm{~K})$, and $\Gamma_{0}=2.12(T>700 \mathrm{~K})$, where $T$ represents the temperature. We solve a system of conservation laws in a two-dimensional Eulerian framework 06 without considering a phase change or cavitation model.

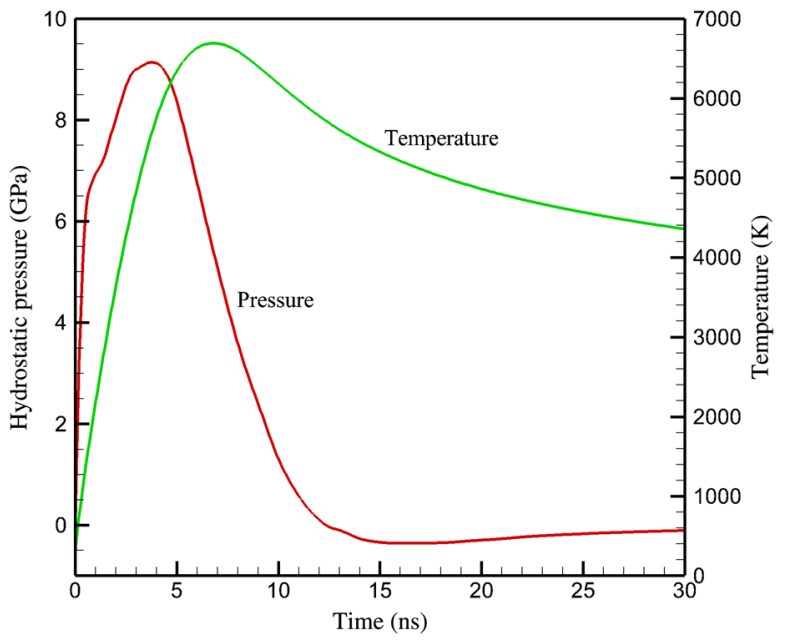

Fig. 7 Time evolution of surface pressure and temperature for $2 \mathrm{~J} / \mathrm{cm}^{2}$ laser pulse.

The energy flux due to the laser irradiation is modeled as a volumetric heat source in the energy equation. Beer's law with a Gaussian distribution describes the absorption of laser energy

$Q(x, y, t)=I_{0}(1-R) \alpha e^{\left(-\alpha\left(y_{s}-y\right)-\frac{\left(x-x_{s}\right)^{2}}{r_{0}^{2}}-\frac{t^{2}}{t_{0}^{2}}\right)}$,

where $\left(x_{s}, y_{s}\right)$ is the surface coordinates of the laser beam center and $z=y_{s}-y$ measures the depth from the surface. Here, the parameters $I_{0}, R, \alpha, t_{0}$, and $r_{0}$ refer to laser energy intensity, reflectance, absorption coefficient, pulse width, and the beam radius, respectively.

In this study, the absorption coefficient is $8.3 \times 10^{7} \mathrm{~m}^{-1}$ and the energy transferred by radiation is not considered. Figure 7 shows the surface pressure and temperature evolution computed at $\left(x_{s}, y_{s}\right)$ for $2 \mathrm{~J} / \mathrm{cm}^{2}$. Our preliminary result shows that pressure is strongly dependent on the value of total incident laser energy density, and the peak pressure value increased with laser energy density. The hydrostatic pressure of $>8 \mathrm{GPa}$ and temperature of $>6000 \mathrm{~K}$ could be generated when laser energy intensity of $2 \mathrm{~J} / \mathrm{cm}^{2}$ laser pulse is used. It should be noted that although SMA surface is not directly irradiated by laser and there is roughly $10 \mu \mathrm{m}$ graphite film between SMA and copper, the high temperature and pressure of the copper surface can still affect SMA surface. The rate of heating or cooling does not affect the phase transformation characteristics. Depending on the temperature and time, second phase formation can be observed in NiTi. However, since equiatomic NiTi was used in this study, the second phase formation is not expected in these alloys. The only effect of rapid heating would be the oxidation of the material surface due to exposed high temperature.

\section{Conclusion}

Aluminum and NiTi SMAs were patterned by advanced direct imprinting method using nanosecond laser pulses with low cost, quick, and low environmental impact. Different templates were successfully imprinted on both aluminum and NiTi. Laser fluence was shown to play a critical role for surface patterning. Laser-induced shock wave imprinting 
was shown to be strong candidate to replace nanoindentation to obtain TWSME.

Controlling the thickness of the depth by varying the laser parameters is a unique feature of laser-assisted shock wave direct imprinting. In addition, no heating or etching is required in this technique. Unlike most direct imprinting techniques, which result in damage to the sample, this technique can produce patterns without damaging the work piece. It is relatively easy to have scalable product and expected to be a competitive technique for advanced manufacturing.

\section{References}

1. K. Sugioka et al., "Ultrafast lasers-reliable tools for advanced materials processing," Eight Sci. App. 3(4), e149 (2014).

2. X. Fei et al., "Surface form memory in NiTi shape memory alloys by laser shock indentation," T. Mat. Sci. 47(5), 2088-2094 (2012).

3. E. Ozbay, "Plasmonics: merging photonics and electronics at nanoscale dimensions," Science 311(5758), 189-193 (2006).

4. N. Shen et al., "Surface micropatterning of pure titanium for biomedical applications via high energy pulse laser peening," t. Micro Nano Manuf. 3(1), 011005 (2015).

5. S. Y. Chou et al., "Imprint of sub-25 nm vias and trenches in polymers," Appl. Phys. Lett. 67(21), 3114-3116 (1995)

6. S. Y. Chou et al., "Imprint lithography with 25 -nanometer resolution," Science 272(5258), 85-87 (1996).

7. L. J. Guo. "Nanoimprint lithography: methods and material requirements," Adv. Mater. 19(4), 495-513 (2007).

8. S. Y. Chou et al., "Sub-10 nm imprint lithography and applications," t. Vac. Sci. Technol. B: Microelectron. Nanometer Struct. Process. Meas. Phenom. 15(6), 2897-2904 (1997).

9. M. Colburn et al., "Patterning nonflat substrates with a low pressure room temperature, imprint lithography process," V. Vac. Sci. Technol. B: Microelectron. Nanometer Struct. 19(6), 2162-2172 (2001).

10. T. Bailey et al., "Step and flash imprint lithography: template surface treatment and defect analysis," P. Vac. Sci. Technol. B: Microelectron. Nanometer Struct. Process. Meas. Phenom. 18(6), 3572-3577 (2000).

11. C. Ye et al., "Scalable patterning on shape memory allow by laser shock assisted imprinting," Appl. Surf. Sci.] 258(24), 10042-10046 (2012).

12. D. J. Leo, Engineering Analysis of Smart Material Systems, John Wiley \& Sons, Hoboken, New Jersey (2007).

13. H. Gao et al., "Large-scale nanoshaping of ultrasmooth 3D crystalline metallic structures," Science 346(6215), 1352-1356 (2014).

14. S. Brady et al., "Numerical study of laser ablation on aluminum for shock-wave applications: development of a suitable model by comparison with recent experiments," Opt. Eng. 56(1), 011014 (2017).

15. Z. Wang et al., "Fabrication of microhole arrays on coated silica sheet using femtosecond laser," Opt. Eng. 55(10), 105101 (2016).

16. R. Fabbro et al., "Physical study of laser-produced plasma in confined geometry," T. Appl. Phys. 68(2), 775-784 (1990).

17. A. H. Clauer et al., "Effects of laser induced shock waves on metals," in Shock Waves and High-Strain-Rate Phenomena in Metals, pp. 675702, Springer, Boston, Massachusetts (1981).

18. B. P. Fairand et al., "Laser shock-induced microstructural and mechanical property changes in 7075 aluminum," T. Appl. Phys. 43(9), 38933895 (1972).

19. L. Hackel et al. "Laser peening to improve fatigue strength and lifetime of critical components 1," Proc. Eng. 133, 545-555 (2015).

20. M. F. El-Kady et al., "Laser scribing of high-performance and flexible graphene-based electrochemical capacitors," Science 335(6074), 13261330 (2012).

21. N. C. Anderholm, "Laser generated pressure waves," Bull. Am. Phys. Soc. 13(3), 388 (1968).

22. N. C. Anderholm, "Laser-generated stress waves," Appl. Phys. Lett. 16, 113-115 (1970).

23. R. E. Wagner, "Laser drilling mechanics," T. Appl. Phys. 45(10), 46314637 (1974)

24. S. H. Niehoff et al., "Laser induced shock waves in deformation processing," Metalurgija 11(3), 183-194 (2005).

25. T. D. Richard, "Laser scribing apparatus," U.S. Patent No. 3,626,141 (1971).

26. A. K. Gujba et al., "Laser peening process and its impact on materials properties in comparison with shot peening and ultrasonic impact peening," Materials 7(12), 7925-7974 (2014).

27. F. J. Carpio et al., "Fatigue behavior of laser machined 2024-T3 aeronautic aluminum alloy," Appl. Surf. Sci. 208-209, 194-198 (2003).

28. M. Z. Ge et al., "Effect of laser shock peening on microstructure and fatigue crack growth rate of AZ31B magnesium alloy," T. Alloys Compd. 680, 544-552 (2016).
29. M. Dorman et al., "Effect of laser shock peening on residual stress and fatigue life of clad 2024 aluminum sheet containing scribe defects," Mater. Sci. Eng. A 548, 142-151 (2012).

30. X. Nie et al., "Experiment investigation on microstructure and mechanical properties of TC17 titanium alloy treated by laser shock peening with different laser fluence," T. Laser Appl. 25(4), 042001 (2013).

31. A. O. Er et al., "Coherent acoustic wave oscillations and melting on $\mathrm{Ag}$ (111) surface by time resolved x-ray diffraction," Appl. Phys. Lett. 100(15), 151910 (2012).

32. A. O. Er et al., "Time-resolved x-ray diffraction studies of laser-induced acoustic wave propagation in bilayer metallic thin crystals," t. Appl. Phys. 116, 093509 (2014).

33. A. O. Er et al., "Ultrafast time resolved x-ray diffraction, extended x-ray absorption fine structure and x-ray absorption near edge structure," T. Appl. Phys. 112, 031101 (2012).

34. F. Gehring et al., "Use of laser shock waves to test piezochromic coatings for impact detection," Opt. Eng. 56(1), 011023 (2017).

35. R. Fabbro et al., "Physics and applications of laser-shock processing," T. Appl. Phys. 10(6), 265-279 (1998).

36. L. Berthe et al., "Wavelength dependent of laser shock-wave generation in the water-confinement regime," T. Appl. Phys. 85(11), 7552-7555 (1999).

37. S. Amoruso et al., "Characterization of laser-ablation plasmas," t.Phys. B: At. Mol. Opt. Phys. 32 (14), R131-R172 (1999).

38. D. B. Chrisey et al., Pulsed Laser Deposition of Thin Films, Wiley, New York (1994).

39. R. Eason, Pulsed Laser Deposition of Thin Films: Applications, John Wiley \& Sons Inc., Hoboken, New Jersey (2007).

40. R. Glaus et al., "Stimulated emission in aluminum laser-induced plasma: an experimental study" Appl. Opt. 56(13), 3699-3702 (2017).

41. P. Peyre et al., "Experimental study of laser-driven shock waves in stainless steels," T. Appl. Phys. 84(11), 5985-5992 (1998).

42. D. C. Lagoudas (Ed.), Shape Memory Alloys: Modeling and Engineering Applications, Springer, New York (2008).

43. K. Otsuka et al., "Recent developments in the research of shape memory alloys," Entermetallics 7(5), 511-528 (1999).

44. D. J. Hartl et al., "Aerospace applications of shape memory alloys," Proc. Inst. Mech. Eng., Part G: J. Aerosp. Eng 221(4), 535-552 (2007)

45. R. D. Noebe et al., "NiTi-based high-temperature shape-memory alloys: properties, prospects, and potential applications," in Advanced Structural Materials, W. O. Soboyejo, Ed., Marcel Dekker, Inc., New York (2006)

46. D. Hyland, "AIAA names top ten emerging aerospace technologies of 2009," 2009, http://intranetaiaa. org/industryresources/PDF/2009Top TenAerospacere Release.pdt

47. L. Gonzalez, "Morphing wing using shape memory alloy: a concept proposal," Final research paper in 2005 Summer Research Experience for Undergraduates (REU) on Nanotechnology and Materials Systems Texas Institute of Intelligent Bio-Nano Materials and Structures for Aerospace Vehicles (TiiMS)-NASA Research University, Texas A\&M University, Texas (2005).

48. C. Thill et al., "Morphing skins," Heronaut. J 112(1129), 117-139 (2008).

49. J. Jimenez et al., "Turbulent flows over rough walls," Ann. Rev. Fluid Mech. 36, 173-196 (2004).

50. D. W. Bechert et al., "Experiments with three-dimensional riblets as an idealized model of shark skin," Exp. Fluids 28(5), 403-412 (2000).

51. F. Klocke et al., "Development of an incremental rolling process for the production of defined riblet surface structures," Prod. Eng. Res. Dev. 1(3), 233-237 (2007).

52. Y.-T. Cheng et al., "Indentation in shape memory alloys." in Micro and Nano Mechanical Testing of Materials and Devices, F. Q. Yang and J. C. M. Li, Eds., pp. 69-84, Springer, Boston, Massachusetts (2008)

53. P. Li et al., "Spherical indentation of NiTi-based shape memory alloys," T. Alloys Compd. 651, 724-730 (2015).

54. Y. Zhang et al., "Shape memory surfaces," Appl. Phys. Lett. 89(4), 041912 (2006).

55. Y. Zhang et al., "Two-way indent depth recovery in a NiTi shape memory alloy," Appl. Phys. Lett. 88(13), 131904 (2006).

56. A. J. Birnbaum et al., "The effects of laser forming on NiTi superelastic shape memory alloys," "T. Manuf. Sci. Eng. 132(4), 041002 (2010).

57. A. S. Turabi et al., "Shape memory effect and superelasticity of NiMnCoIn metamagnetic shape memory alloys under high magnetic field," Script. Mater. 111, 110-113 (2016).

58. H. E. Karaca et al., "Stress-induced martensite to austenite phase transformation in Ni2MnGa magnetic shape memory alloys," Smart Mater. Struct. 21(4), 045011 (2012).

59. H. E. Karaca et al., "NiTiHf-based shape memory alloys," Mater. Sci. Technol. 30(13), 1530-1544 (2014).

60. H. E. Karaca et al., "Compressive response of polycrystalline NiCoMnGa high-temperature meta-magnetic shape memory alloys," J. Mater. Eng. Perform. 22, 3111-3114 (2013). 
61. P. Li et al., "Rapid characterization of local shape memory properties through indentation," Sci. Rep. 7, 14827 (2017).

62. $\mathrm{P}$. $\mathrm{Li}$ et al., "Orientation dependent compression behavior of Co35Ni35Al30 single crystals," t. Alloys Compd. 718, 326-334, (2017).

63. M. S Brown et al.. "Fundamentals of laser-material interaction and application to multiscale surface modification," Laser Precis. Microfab 135, 91-120 (2010)

64. O. San et al., "Evaluation of Riemann flux solver for WENO reconstruction schemes: Kelvin-Helmholtz instability," Comput. Fluids 117, 24-41 (2015).
65. V. A. Titarev et al., "MUSTA-type upwind fluxes for non-linear elasticity," Int. J. Numer. Meth. Eng. 73(7), 897-926 (2008).

66. B. Mehmandoust et al., "An Eulerian particle level set method for compressible deforming solids with arbitrary EOS," Int. J. Num. Meth. Eng. 79(10), 1175-1202 (2009).

67. S. Ilhom et al., "Laser shock wave-assisted patterning on NiTi shape memory alloy surfaces," Shap. Mem. Superelasticity 1-8 (2018).

68. D. Seyitliev et al., "Laser-induced hydrogen generation from graphite and coal," Int. J. Hydrogen Energy 42, 26277-26288 (2017).

Biographies for the authors are not available. 